\title{
Esteatose pancreática
}

José Galvão-Alves

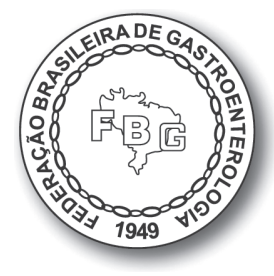

Bruna Cerbino de Souza

\section{RESUMO}

A prevalência da obesidade vem aumentando exponencialmente em todo o mundo, sendo considerada hoje uma epidemia global. Relaciona-se, especialmente em seu tipo central, com o desenvolvimento de resistência insulínica e infiltração gordurosa de vários órgãos, como coração, rins, fígado e pâncreas. Bem avaliados e demonstrados são os efeitos do acúmulo de gordura visceral e suas consequências cardiometabólicas, no entanto, o pâncreas constitui um sítio de depósito ectópico ainda pouco explorado. No passado, a esteatose pancreática era encarada como uma condição inocente ou apenas parte das manifestações clínicas da obesidade. Atualmente, recentes pesquisas sugerem sua relação com disfunção e inflamação das células beta, maior gravidade dos quadros de pancreatite, risco aumentado de fístulas pós-operatórias e, eventualmente, fator contribuinte para o adenocarcinoma de pâncreas.

O objetivo deste capítulo é reunir o que há de mais moderno na literatura mundial sobre potencial fisiopatologia, métodos diagnósticos e implicações clínicas da esteatose pancreática, além de suscitar o desenvolvimento de novos estudos para melhor análise e documentação de seus efeitos.

\section{INTRODUÇÃO}

A obesidade é atualmente considerada um dos maiores problemas de saúde pública no mundo, e sua incidência vem aumentando a cada década, em todas as faixas etárias. A Organização Mundial da Saúde (OMS) estima que, em 2017, mais de 1,9 bilhão de adultos apresentavam sobrepeso e, destes, 650 
milhões eram obesos. A prevalência da obesidade quase triplicou entre 1975 e 2017. Em 2016, 41 milhões de crianças abaixo de 5 anos tinham sobrepeso ou eram obesas ${ }^{1}$.

Nos últimos anos, o conceito de que a obesidade não é uma condição homogênea vem se consolidando, resultado da descoberta de que a distribuição locorregional do tecido adiposo influencia de modos distintos os metabolismos glicídico e lipídico, gerando um desequilíbrio nas reações anabólicas e catabólicas do organismo, além de disbiose $e^{2-4}$.

Quando os níveis séricos de triglicerídios e ácidos graxos livres excedem a capacidade metabólica do indivíduo, estes se depositam em tecidos não adiposos, como músculo esquelético, fígado, rins, coração, suprarrenais e pâncreas.

Essa última disposição, a esteatose pancreática, foi primeiramente descrita em 1933 por Ogilvie ${ }^{5}$. Em sua pesquisa, observou em cadáveres que 9\% daqueles que eram magros apresentavam acúmulo de gordura pancreática em comparação a $17 \%$ encontrados em obesos. Olsen et al. constataram que a concentração de gordura pancreática aumenta substancialmente com a idade ${ }^{6}$, e Stamm encontrou significativamente mais diabetes melito tipo 2 e aterosclerose naqueles que apresentavam mais de $25 \%$ de gordura pancreática ${ }^{7}$.

Nos dias atuais, a deposição de lipídios no pâncreas vem ganhando maior importância, pela sugestiva correlação com o desenvolvimento de resistência insulínica, inflamação celular e lipotoxicidade, que se acredita serem responsáveis pelos transtornos glicometabólicos e demais alterações apresentadas por estes pacientes, as quais serão abordadas neste trabalho.

\section{NOMENCLATURA}

Muitos sinônimos para designar o acúmulo de gordura no tecido pancreático podem ser encontrados na literatura, gerando, por vezes, confusão entre as diferentes situações clínicas. Smits e van Geenen propuseram, em recente artigo, uma padronização para orientar o emprego dos diferentes termos ${ }^{8}$. Esteatose é um nome genérico utilizado para discriminar o depósito de gordura no interior das células parenquimatosas. Assim, esteatose pancreática descreveria o acúmulo de gordura nas células das ilhotas ou ácinos e também no interior dos adipócitos. Na opinião dos autores, o termo substituição gordurosa deve ter seu uso restrito aos casos em que há dano permanente às células acinares, levando-as à morte e a posterior substituição por adipócitos. Infiltração gordurosa foi substituída pelo termo doença pancreática gordurosa não alcoólica (DPGNA), à semelhança do processo que se desenvolve no fígado. Essa descrição tem seu emprego justificado nos casos de esteatose pancreática como consequência da obesidade e síndrome metabólica e na ausência da ingesta habitual de bebidas 
alcoólicas. O termo lipomatose pancreática é utilizado como sinônimo para substituição gordurosa do tecido exócrino, enquanto o termo pseudo-hipertrofia lipomatosa é usado para designar o pâncreas aumentado de volume, com seus ácinos substituídos por gordura, mas no qual não se encontra nenhuma relação com obesidade. Entretanto, a esteatose pancreática também se relaciona ao aumento do volume pancreático e nem sempre está relacionada à obesidade, o que leva a acreditar, na opinião dos autores, que a pseudo-hipertrofia lipomatosa seria uma condição extrema de deposição de gordura que, todavia, não se dissocia classicamente da esteatose (Tabela 1).

\begin{tabular}{|c|c|}
\hline Nomenclatura & Definição \\
\hline $\begin{array}{l}\text { Lipomatose pancreática } \\
\text { Esteatose pancreática }\end{array}$ & $\begin{array}{l}\text { Termos gerais que podem ser empregados para todas } \\
\text { as formas de acúmulo de gordura pancreática }\end{array}$ \\
\hline Pseudo-hipertrofia lipomatosa & $\begin{array}{l}\text { Variante extrema na qual o pâncreas encontra- } \\
\text {-se aumentado de volume, os ácinos sofrem } \\
\text { lipossubstituição e nenhuma associação com } \\
\text { obesidade é encontrada }\end{array}$ \\
\hline Substituição gordurosa & $\begin{array}{l}\text { Dano às células pancreáticas acinares que resulta em } \\
\text { morte delas e posterior substituição por adipócitos } \\
\text { (em geral, irreversível) }\end{array}$ \\
\hline Infiltração gordurosa & $\begin{array}{l}\text { Infiltração pancreática por adipócitos causada pela } \\
\text { obesidade (possivelmente reversível com redução do } \\
\text { peso corporal e medicações apropriadas) }\end{array}$ \\
\hline $\begin{array}{l}\text { Doença pancreática gordurosa não } \\
\text { alcoólica }\end{array}$ & $\begin{array}{l}\text { Deposição de gordura pancreática relacionada com } \\
\text { obesidade e síndrome metabólica }\end{array}$ \\
\hline $\begin{array}{l}\text { Esteatopancreatite gordurosa não } \\
\text { alcoólica }\end{array}$ & $\begin{array}{l}\text { Pancreatite decorrente de acúmulo de gordura } \\
\text { pancreática }\end{array}$ \\
\hline
\end{tabular}

\section{PATOGENIA}

Macrófagos são células que participam da resposta imune inata do organismo, altamente dinâmicas, alternando entre os estados anti-inflamatório (M2) e pró-inflamatório (M1) nos tecidos residentes com facilidade. No indivíduo magro, encontram-se, em seu tecido adiposo, células natural killer (NK) e macrófagos do tipo M2, responsáveis pela secreção de interleucina-10 (IL-10), a qual promove a manutenção do estado anti-inflamatório e da sensibilidade à insulina ${ }^{9}$. A obesidade se caracteriza pela ativação crônica das vias inflamatórias, por meio da estimulação da lipólise e posterior liberação de ácidos graxos 
livres e fatores pró-inflamatórios. No tecido adiposo dos indivíduos obesos, tais mediadores pró-inflamatórios, como o TNF-alfa, recrutam monócitos que serão futuramente transformados em macrófagos do tipo $\mathrm{M} 1^{10}$. Esse padrão celular destaca-se por ser altamente pró-inflamatório e, quando ativado, induz a produção de outros sinalizadores, como interleucina-1-beta, IL-6, leucotrieno B4 e óxido nítrico, capazes de manter o estado inflamatório e, de forma parácrina, levar à resistência insulínica ${ }^{11}$. $\mathrm{O}$ excesso de ácidos graxos livres deposita-se em outros sítios, como fígado, músculo estriado e pâncreas, além de ser responsável pela indução de sinais inflamatórios nestes locais e também contribuir para a resistência insulínica. No fígado, o acúmulo de lipídios gera o estresse crônico do retículo endoplasmático dos hepatócitos, além da conversão dos macrófagos, denominados células de Kupffer, em um fenótipo M1, determinando a produção de citocinas inflamatórias, oncostatina e prostaglandinas ${ }^{12-14}$. Tais moléculas sinalizadoras e os ácidos graxos livres liberados pelo tecido adiposo inflamado no estado de obesidade, assim como o conteúdo resultante do metabolismo da microbiota intestinal alterada, contribuem para a polarização das células de Kupffer em um status inflamatório constante e perpetuado, ocasionando a resistência insulínica hepática e o desenvolvimento de DHGNA ${ }^{15}$.

Em razão da origem embriológica semelhante compartilhada entre fígado e pâncreas, acredita-se que possam existir etapas comuns no processo de deposição de gordura ectópica nestes órgãos. É frequente observar a coexistência de DPGNA e DHGNA, e ambas as condições estão fortemente associadas à obesidade e ao acúmulo de gordura visceral ${ }^{16,17}$.

Assim, dois potenciais mecanismos podem levar à esteatose pancreática: morte das células acinares, seguida pela substituição destas por tecido adiposo; e depósito intracelular de triglicerídios relacionado com um excessivo balanço energético ${ }^{8}$. Há uma relação entre maior ingesta calórica e aumento do tecido adiposo visceral e subsequente acúmulo de gordura ectópica. Em ratos alimentados com dieta hiperlipídica para indução da obesidade, foram observados hipertrofia dos adipócitos, esteatose hepatopancreática e intolerância à glicose. Notou-se ainda que o peso do pâncreas foi maior nos obesos quando comparado aos ratos magros. Além disso, as citocinas pró-inflamatórias IL-1-beta e TNF-alfa também estavam significativamente elevadas no pâncreas dos ratos obesos ${ }^{16}$.

Considerando todos estes achados, é possível inferir que a esteatose pancreática é consequência do excesso de lipídios decorrente de um desequilíbrio no balanço energético corporal, e seu desenvolvimento é mediado, em parte, pelo status inflamatório crônico ocasionado pela obesidade. Novos estudos são necessários para melhor elucidar a inter-relação entre os processos imunomediados e sua base molecular. 


\section{DIAGNÓSTICO}

\section{Histologia}

Análises de amostras de tecido pancreático à microscopia revelam a presença de numerosos adipócitos. Com o auxílio da imuno-histoquímica, é possível visualizar o acúmulo intracelular de gordura nos ácinos e nas ilhotas, o qual precede a infiltração do parênquima por adipócitos, segundo Lee et al. ${ }^{18}$.

Imagem

Atualmente, a avaliação e a quantificação do acúmulo de gordura pancreática são feitas de forma não invasiva, por meio de diferentes exames de imagem, como ultrassonografia, tomografia computadorizada (TC) e ressonância magnética (RM). Todos visam à graduação indireta da infiltração gordurosa no pâncreas, objetivando encontrar resultados aproximados aos da análise histológica, cujas amostras são obtidas via biópsia, considerada o padrão-ouro no diagnóstico desta condição. A esteatose pancreática é ainda, com certa frequência, um achado incidental nos exames de imagem. À ultrassonografia, apresenta-se como uma hiperecogenicidade do tecido pancreático ${ }^{19,20}$. No entanto, a fibrose do parênquima também se mostra hiperecogênica sob esta técnica, o que, de certa forma, restringe seu emprego no rastreamento da esteato$\mathrm{se}^{20,21}$. Também é limitante o fato de que, sobretudo nos indivíduos obesos, o pâncreas não é bem ou completamente visualizado à ultrassonografia. A detecção da esteatose pela ultrassonografia utiliza a comparação entre a ecogenicidade do pâncreas com aquela exibida pelo fígado e pelos rins; uma ecogenicidade pancreática maior apontaria para a presença de gordura parenquimatosa, enquanto uma densidade semelhante à exibida pela gordura retroperitoneal indicaria maior severidade do quadro ${ }^{22}$.

A análise do pâncreas esteatótico sob TC revela um parênquima de densidade análoga à do tecido adiposo (Figuras 1 e 2). Trata-se de um exame rápido, amplamente disponível, que dispensa o emprego de contraste venoso para o diagnóstico da esteatose pancreática. No entanto, utiliza radiação ionizante, não tem sensibilidade adequada para a detecção dos casos leves de infiltração gordurosa focal e ainda não há limites definidos para o diagnóstico da esteatose pancreática ${ }^{23}$.

À RM, a gordura macroscópica apresenta-se hiperintensa, tanto nas sequências ponderadas em T1 quanto em T2 (Figuras 3 e 4). O gradiente eco (GRE) com desvio químico em "fase" e "oposição de fase" é utilizado na ava- 


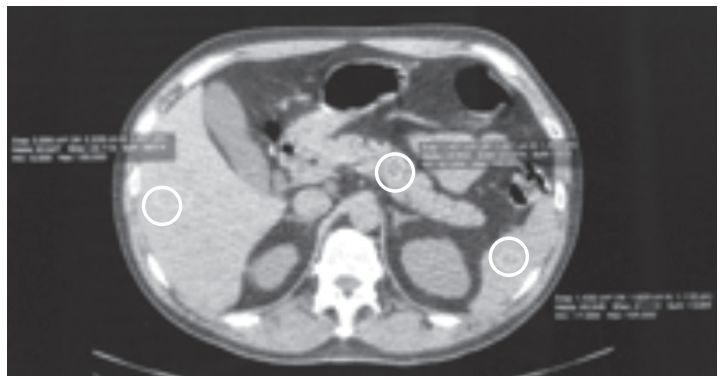

Figura 1 Tomografia computadorizada de abdome sem contraste - tecido análogo ao tecido adiposo.

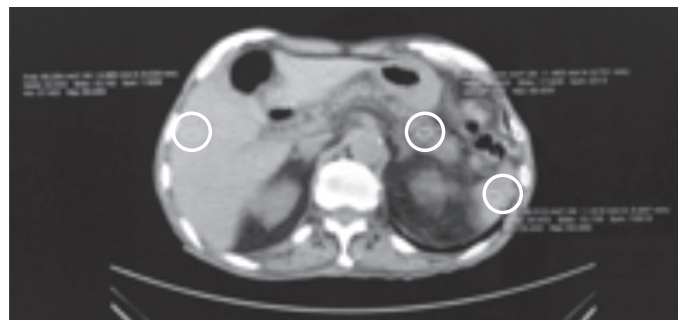

Figura 2 Tomografia computadorizada de abdome sem contraste - tecido análogo ao adiposo.

liação de gordura intracelular que, quando presente, provoca uma perda de intensidade de sinal nas imagens obtidas em "oposição de fase" comparativamente às obtidas em "fase" 24,25 . Tal perda de sinal é proporcional à quantidade de gordura, sendo este um método de elevada acurácia na quantificação de gordura pancreática, quase equivalente à histologia e à bioquímica para uso in vivo.

A ultrassonografia endoscópica (USE) surge como nova modalidade para o diagnóstico da esteatose pancreática. Em virtude da proximidade da sonda com o tecido pancreático, exibe resolução espacial superior àquelas apresentadas pela TC e pela RM, além de possibilitar a coleta de amostras do parênquima pancreático (Figuras 5 a 7 ) $^{19}$. Contudo, é um procedimento invasivo, que requer sedação e que apresenta alguns riscos de complicação, embora estes sejam pequenos.

\section{ETIOLOGIA}

Como já discutido, a obesidade tem um papel crucial na gênese da esteatose pancreática, resultando na infiltração do tecido pancreático por adipócitos. 


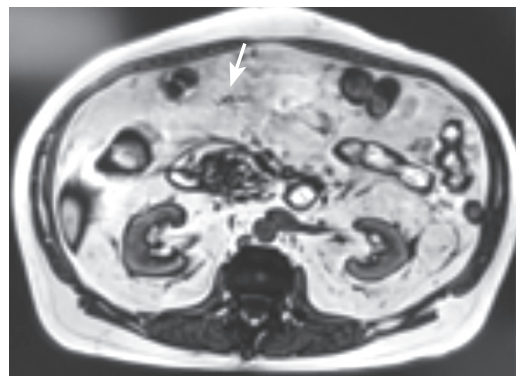

Figura 3 Ressonância magnética: método não invasivo de elevada acurácia.

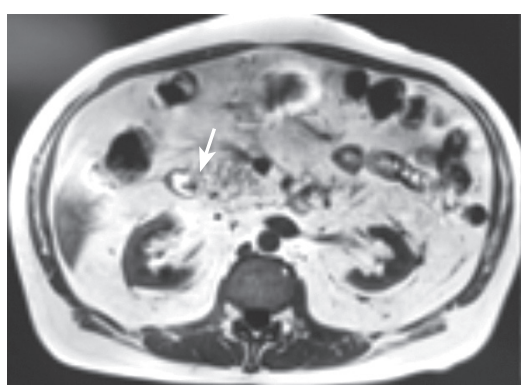

Figura 4 Ressonância magnética: método não invasivo de elevada acurácia.

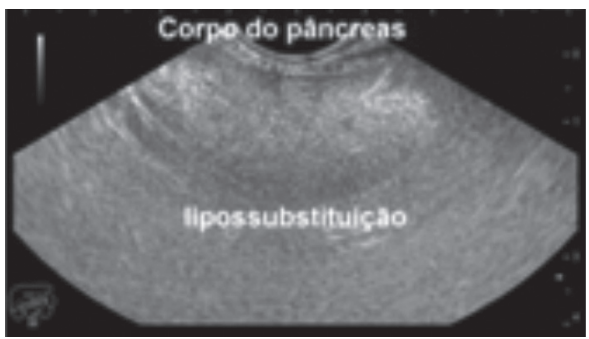

Figura 5 Ultrassonografia endoscópica: método superior a tomografia computadorizada/ressonância magnética; permite biópsia do tecido pancreático.

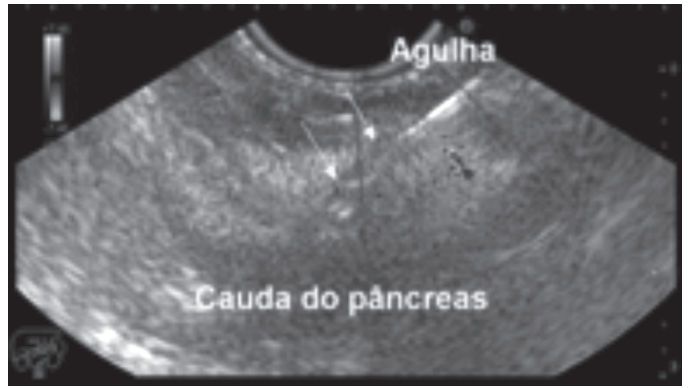

Figura 6 Biópsia do tecido pancreático. 


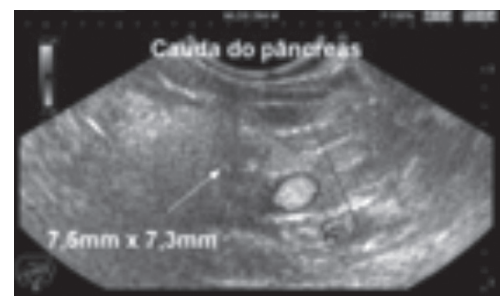

Figura 7 Ecoendoscopia: permite análise mais detalhada e precisa do tecido pancreático.

Além disso, a idade avançada também vem sendo associada à maior densidade lipídica do pâncreas. No indivíduo jovem, a quantidade de gordura aumenta proporcionalmente ao volume do parênquima pancreático. Já nos idosos, essa deposição não se guia pelo aumento volumétrico do pâncreas. No entanto, estas não são as únicas condições responsáveis pelo depósito pancreático de gordura, podendo este ser causado por diabetes melito, síndromes congênitas, infecções e estados de desnutrição ${ }^{26-33}$ (Tabela 2).

Tabela 2 Etiologia da esteatose pancreática

Idade avançada

Obesidade

Diabetes melito

Consumo excessivo de álcool (> $140 \mathrm{~g} / \mathrm{semana}$ )

Hemocromatose

Infecções virais: hepatite B, reovírus, vírus da imunodeficiência humana e síndrome da imunodeficiência adquirida (HIV/aids)

Desnutrição: Kwashiorkor

Medicamentos: gencitabina e rosiglitazona

Síndromes congênitas: fibrose cística, síndrome de Shwachman-Diamond, síndrome de Johanson-Blizzard, mutações heterozigotas da carboxil-ester-lipase

Fonte: adaptada de Tariq et al., $2016^{34}$.

\section{CONSEQUÊNCIAS CLÍNICAS}

Síndrome metabólica e diabetes melito

A síndrome metabólica engloba um conjunto de fatores de risco metabólico que elevam as chances de um indivíduo desenvolver diabetes ou complicações cardiovasculares. Seus critérios diagnósticos incluem: obesidade central, 
intolerância à glicose ou diabetes já estabelecido, hipertensão arterial e dislipidemia; 3 ou mais destes itens fecham o diagnóstico. Sepe et al. encontraram uma alta prevalência de esteatose pancreática nos indivíduos que apresentavam síndrome metabólica e estimaram que a presença de qualquer um dos componentes da síndrome aumenta a ocorrência de esteatose em $37 \%{ }^{35}$. Wu et al. confirmaram em seus estudos que a esteatose pancreática está fortemente relacionada com parâmetros da síndrome metabólica ${ }^{36}$. Deduz-se, então, que o acúmulo de gordura pancreática pode representar uma das formas de expressão da síndrome, e alguns pesquisadores já sugerem que a DPGNA seja incorporada aos critérios diagnósticos deste distúrbio ${ }^{37}$.

A resistência insulínica é considerada fator determinante e compartilhado no estabelecimento da DPGNA e diabetes, no entanto, a relação entre essas condições clínicas e sua cronologia ainda é controversa.

Vários estudos têm proposto uma potencial associação entre disglicemia, esteatose pancreática e disfunção das células beta. A explicação mais comumente aceita é a de que, nas células beta pancreáticas, a hiperglicemia inibiria a carnitina parmitoil transferase I, por meio do aumento da malonil coenzima A, reduzindo a betaoxidação mitocondrial e promovendo a deposição intracelular de triglicerídios. A resistência insulínica ocasionaria ainda a diminuição da ação inibitória da insulina sobre a lipólise nos tecidos periféricos, produzindo maior quantidade de ácidos graxos circulantes. A exposição crônica das células beta a tais ácidos graxos livres resultaria no aumento do teor de triglicerídios, diminuição da expressão do gene da insulina e maior risco de apoptose das células beta ${ }^{38}$. O efeito deletério da glicolipotoxicidade sobre as células das ilhotas pancreáticas parece favorecer sua disfunção, além de acelerar o estabelecimento do diabetes melito nos indivíduos predispostos, visto que promove a manutenção de um ciclo vicioso de deterioração contínua do estado glicometabólico ${ }^{39}$. Outra tese é que os adipócitos pancreáticos exerçam um efeito negativo parácrino sobre as células beta.

Wang et al. observaram um aumento significativo de diabetes melito tipo 2 em pacientes com DPGNA, em comparação com os indivíduos controle saudáveis $(12,6$ vs. 5,2\%, $\mathrm{P}<0,001$, respectivamente). A análise de regressão revelou que a presença de esteatose pancreática estava associada com obesidade central, DHGNA e diabetes $(\mathrm{P}<0,001)^{40}$.

Corte et al. avaliou o efeito metabólico da esteatose pancreática em uma população pediátrica obesa, constatando que havia uma associação positiva entre os níveis de resistência insulínica e DPGNA ${ }^{41}$.

Em contraste, estudo recente conduzido por Saisho et al. não encontrou uma relação causal entre esteatose pancreática e diabetes ${ }^{42}$, resultado confir- 
mado por van der Zijl et al., que não evidenciaram correlação entre DPGNA e hiperglicemia decorrente de um padrão de secreção pancreática alterado ${ }^{43}$.

Sabe-se atualmente que a esteatose pancreática pode estar presente na fase pré-diabética e que, após o estabelecimento do DM, essa condição se agrava. No entanto, até o momento, o que se observou é que a DPGNA e o diabetes melito tipo 2 são consequências da obesidade, não sendo possível afirmar que a esteatose pancreática desempenha algum papel na patogênese do diabetes melito tipo 2, sendo necessárias novas pesquisas para comprovar ou mesmo refutar essa inter-relação.

\section{Insuficiência exócrina pancreática}

É bem estabelecido na literatura que, para a ocorrência de sinais e sintomas relacionados à insuficiência exócrina pancreática (IEP), deve haver um grande comprometimento das células acinares. Entretanto, Dupont et al. teorizaram que a redução na secreção enzimática é um fenômeno precoce na lipomatose pancreática, e que esta seria a $2^{\text {a }}$ causa mais frequente de insuficiência exócrina na população pediátrica ${ }^{44}$. De fato, em algumas síndromes congênitas (fibrose cística, síndrome de Shwachman-Diamond e síndrome de Johanson-Blizzard), há, com alguma frequência, a coexistência de IEP e esteatose pancreática, porém, dados científicos acerca do tema são escassos e não permitem inferir uma relação causal para a população em geral. Alguns relatos de caso ${ }^{45-48}$ propõem uma associação com insuficiência exócrina e, portanto, novos estudos prospectivos devem ser realizados para determinar o real impacto da esteatose no pâncreas exócrino.

\section{Pancreatite aguda}

O risco para o desenvolvimento de pancreatite aguda é maior nos pacientes obesos e com síndrome metabólica e está relacionado aos seguintes fatores:

- Presença de colelitíase, seja pela maior formação de cálculos de colesterol decorrente da dieta hiperlipídica ou pela estase da vesícula biliar gerado por intervalos maiores entre as refeições como tentativa de redução do peso corporal.

- Hipertrigliceridemia, cujo excesso de triglicerídios insolúveis no ambiente aquoso sanguíneo resulta em microtrombos na vasculatura pancreática, causando infarto e isquemia do órgão.

- Efeito adverso das medicações utilizadas no diabetes/síndrome metabólica, como peptídio semelhante a glucagon 1 (GLP-1) e inibidores da dipeptidilpeptidase (DDP-4) ${ }^{49}$. 
Tagushi et al. relataram maior risco relativo de morte nos quadros de pancreatite aguda em obesos quando comparado com controles de peso normal (RR 6,4; intervalo de confiança de 95\%, 1,9-20,9) ${ }^{50}$.

Estudos recentes têm demonstrado um aumento na resposta inflamatória nos pacientes obesos, orquestrada pelos adipócitos e mediada por citocinas pró-inflamatórias como leptina, IL-6, IL-1-beta e TNF-alfa, que facilitam a ocorrência de pancreatite aguda. Outro estudo evidenciou uma correlação significativa entre o grau de esteatose pancreática e a gravidade do quadro de pancreatite ${ }^{8,51}$.

A esteatose pancreática pode ser uma condição relacionada à maior gravidade dos quadros de pancreatite aguda, embora não seja fator causal para esta última.

\section{Câncer de pâncreas}

Obesidade, especialmente a forma central, é considerada um fator de risco bem definido para o câncer de pâncreas, assim como tabagismo, alcoolismo, história familiar, diabetes e pancreatite crônica. Stolzenberg et al. afirmaram haver um risco acima de $45 \%$ para o desenvolvimento de câncer de pâncreas em indivíduos com IMC $>35^{52}$.

Atualmente, novos estudos têm revelado uma possível contribuição da infiltração gordurosa do tecido pancreático na gênese do adenocarcinoma deste órgão $0^{53}$.

Recentes pesquisas sugerem que o depósito de gordura no pâncreas acarrete a produção de adipocinas por estes adipócitos e que, quando associadas a outros fatores predisponentes encontrados no estado de obesidade, como estresse oxidativo e resistência insulínica, favoreçam a ocorrência de neopla$\operatorname{sia}^{54,55}$. A presença de esteatose pancreática parece promover ainda uma maior progressão e propagação do tumor, segundo dados recentes ${ }^{56}$.

Acredita-se que, à semelhança do que ocorre no fígado, o acúmulo de gordura pancreática possa levar a um estado pró-inflamatório crônico, que, por sua vez, ocasiona episódios repetidos de pancreatite aguda, até que as alterações características de pancreatite crônica se estabelecem e, em resposta a ela, o adenocarcinoma se desenvolve.

\section{Fístulas pancreáticas}

Duodenopancreatectomia pode complicar com a ocorrência de fístulas pancreáticas, cujo risco se relaciona com a consistência macia do tecido pancreático remanescente ${ }^{57}$. Mathur et al. estabeleceram um estudo pioneiro que constatou que a presença de esteatose pancreática aumenta significativamente o risco de surgimento de fístulas pós-operatórias $(\mathrm{p}<0,001)^{58}$. 
Outras pesquisas confirmaram essa inter-relação $0^{59}$, e Lee et al. concluíram que a consistência do pâncreas está intimamente relacionada com a quantidade de gordura depositada nele ${ }^{60}$. Além disso, um estudo mostrou que indivíduos que possuíam $10 \%$ de tecido adiposo no pâncreas apresentavam uma maior chance de complicações pós-cirúrgicas, como deiscência de anastomose e fístulas pancreáticas ${ }^{61}$.

\section{CONSIDERAÇÕES FINAIS}

Após revisão da literatura, conclui-se que a esteatose pancreática tem uma associação significativa com a obesidade e a síndrome metabólica. Os componentes desta síndrome provocam alterações metabólicas, como resistência insulínica, inflamação tissular e estresse oxidativo, que contribuem para o surgimento, o agravo e a progressão desta situação clínica. Embora facilmente detectada pelos exames de imagem, poucos estudos sobre a esteatose pancreática foram desenvolvidos até o momento, e o que outrora era considerada uma condição inócua, hoje parece apresentar implicações clínicas expressivas.

Novas pesquisas são necessárias para o estabelecimento de critérios clínicos, patológicos e radiológicos, a fim de instituir normas padronizadas para o diagnóstico e a classificação dos diferentes estágios da doença, estratégias para seu tratamento e de suas complicações de longo prazo.

\section{REFERÊNCIAS BIBLIOGRÁFICAS}

1. World Health Organization (WHO). Obesity and overweight, 2017. Disponível em: www.who.int/ mediacentre/factsheets/fs311/en/.

2. Wajchenberg BL. Subcutaneous and visceral adipose tissue: their relation to the metabolic syndrome. Endocr Rev. 2000;21:697-738.

3. Vague J. La différenciation sexuelle; facteur determinant desformes de l'obésité. Presse Med. 1947;55(30):339.

4. Bouchard C, Despres JP, Mauriege P. Genetic and nongenetic determinants of regional fat distribution. Endocr Rev. 1993;14:72-93.

5. Ogilvie R. The island of Langerhans in 19 cases of obesity. J Pathol. 1933;37:473-81.

6. Olsen TS. Lipomatosis of the pancreas in autopsy material and its relation to age and overweight. Acta Pathol Microbiol Scand 1978;86a:367-73.

7. Stamm BH. Incidence and diagnostic significance of minor pathologic changes in the adult pancreas at autopsy: a systematic study of 112 autopsies in patients without known pancreatic disease. Hum Pathol. 1984;15:677-83.

8. Smits MM, van Geenen EJM. Nat Rev Gastroenterol Hepatol. 2011;8:169-77.

9. Nguyen MT, Satoh H, Favelyukis S, Babendure JL, Imamura T, Sbodio JI, et al. JNK and tumor necrosis factor-alpha mediate free fatty acid-induced insulin resistance in 3T3-L1 adipocytes. J Biol Chem. 2005;280(42):35361-71.

10. McNelis JC, Olefsky JM. Macrophages, immunity, and metabolic disease. Immunity. 2014;41(1):36-48. 
11. Suganami T, Nishida J, Ogawa Y. A paracrine loop between adipocytes and macrophages aggravates inflammatory changes: role of free fatty acids and tumor necrosis factor alpha. Arterioscler Thromb Vasc Biol. 2005;25(10):2062-8.

12. Dixon LJ, Barnes M, Tang H, Pritchard MT, Nagy LE. Kupffer cells in the liver. Compr Physiol. 2013;3(2):785-97.

13. Jager J, Aparicio-Vergara M, Aouadi M. Liver innate immune cells and insulin resistance: the multiple facets of Kupffer cells. J Intern Med. 2016;280(2):209-20.

14. Kolios G, Valatas V, Kouroumalis E. Role of Kupffer cells in the pathogenesis of liver disease. World J Gastroenterol. 2006;12(46):7413-20.

15. Henkel J, Gartner D, Dorn C, Hellerbrand C, Schanze N, Elz SR, et al. Oncostatin M produced in Kupffer cells in response to PGE2: possible contributor to hepatic insulin resistance and steatosis. Lab Invest. 2011;91(7):1107-17.

16. Fraulob JC, Ogg-Diamantino R, Fernandes-Santos C, Aguila MB, Mandarim-de-Lacerda CA. A mouse model of metabolic syndrome: insulin resistance, fatty liver and non-alcoholic fatty pancreas disease (NAFPD) in C57BL/6 mice fed a high fat diet. J Clin Biochem Nutr. 2010;46(3):212-23.

17. van Geenen EJ, Smits MM, Schreuder TC, van der Peet DL, Bloemena E, Mulder CJ. Nonalcoholic fatty liver disease is related to nonalcoholic fatty pancreas disease. Pancreas. 2010;39(8):1185-90.

18. Lee JS, Kim SH, Jun DW, Han JH, Jang EC, Park JY, et al. Clinical implications of fatty pancreas: correlations between fatty pancreas and metabolic syndrome. World J Gastroenterol. 2009;15(15):1869-75.

19. Al-Haddad M, Khashab M, Zyromski N, Pungpapong S, Wallace MB, Scolapio J, et al. Risk factors for hyperechogenic pancreas on endoscopic ultrasound: a case-control study. Pancreas. 2009;38(6):672-5.

20. Schneider K, Harms K, Fendel H. The increased echogenicity of the pancreas in infants and children: the white pancreas. Eur J Pediatr. 1987;146:508-11.

21. Shawker TH, Linzer M, Hubbard VS. Chronic pancreatitis: the diagnostic significance of pancreatic size and echo amplitude. J Ultrasound Med. 1984;3:267-72.

22. Worthen NJ, Beabeau D. Normal pancreatic echogenicity: relation to age and body fat. AJR Am J Roentgenol. 1982;139:1095-8.

23. Katz DS, Hines J, Math KR, Nardi PM, Mindelzun RE, Lane MJ. Using CT to reveal fat containing abnormalities of the pancreas. AJR Am J Roentgenol. 1999;172(2):393-6.

24. Kim HJ, Byun JH, Park SH, Shin YM, Kim PN, Ha HK, et al. Focal fatty replacement of the pancreas: usefulness of chemical shift MRI. AJR Am J Roentgenol. 2007;188(2):429-32.

25. Kovanlikaya A, Mittelman SD, Ward A, Geffner ME, Dorey F, Gilsanz V. Obesity and fat quantification in lean tissues using three-point Dixon MR imaging. Pediatr Radiol. 2005;35(6):601-7.

26. Jones JS. Adult cystic fibrosis (mucoviscidosis). Fatty replacement of the pancreas in a woman aged 47 years. Br J Dis Chest. 1970;64(1):25-36.

27. Cubuk M, Arslan G, Ceken K, Ozkaynak C, Luyleci E. Schwachman-Diamond syndrome. A case report. Acta Radiol. 2000;41(6):627-8.

28. Lee JH, Bae SH, Yu JJ, Lee R, Yun YM, Song EY. A case of Shwachman-Diamond syndrome confirmed with genetic analysis in a Korean child. J Korean Med Sci. 2008;23(1):142-5.

29. Daentl DL, Frias JL, Gilbert EF, Opitz JM. The Johanson-Blizzard syndrome: case report and autopsy findings. Am J Med Genet. 1979;3(2):129-35.

30. Raeder H, Haldorsen IS, Ersland L, Gruner R, Taxt T, Sovik O, et al. Pancreatic lipomatosis is a structural marker in nondiabetic children with mutations in carboxyl-ester lipase. Diabetes. 2007;56(2):444-9.

31. Sasaki M, Nakanuma Y, Ando H. Lipomatous pseudohypertrophy of the pancreas in a patient with cirrhosis due to chronic hepatitis B. Pathol Int. 1998;48(7):566-8.

32. Diamond I, Vallbona C. Kwashiorkor in a North American white male. Pediatrics. 1960;25:248-57. 
33. Pezzilli R, Calculli L. Pancreatic steatosis: is it related to either obesity or diabetes mellitus? World J Diabetes. 2014;5(4):415-9.

34. Tariq H, Nayudu S, Akella S, Glandt M, Chilimuri S. Non-alcoholic fatty pancreatic disease: a review of literature. Gastroenterol Res. 2016;9(6):87-91.

35. Sepe PS, Ohri A, Sanaka S, Berzin TM, Sekhon S, Bennett G, et al. A prospective evaluation of fatty pancreas by using EUS. Gastrointest Endosc. 2011;73(5):987-93.

36. Wu WC, Wang CY. Association between non-alcoholic fatty pancreatic disease (NAFPD) and the metabolic syndrome: case-control retrospective study. Cardiovasc Diabetol. 2013;12:77.

37. Musso G, Gambino R, Bo S, Uberti B, Biroli G, Pagano G, et al. Should nonalcoholic fatty liver disease be included in the definition of metabolic syndrome? A cross-sectional comparison with Adult Treatment Panel III criteria in nonobese nondiabetic subjects. Diabetes Care. 2008;31(3):562-8.

38. Zhang X, Cui Y, Fang L, Li F. Chronic high-fat diets induce oxide injuries and fibrogenesis of pancreatic cells in rats. Pancreas. 2008;37(3):e31-e38.

39. Poitout V, Amyot J, Semache M, Zarrouki B, Hagman D, Fontés G. Glucolipotoxicity of the pancreatic beta cell. Biochim Biophys Acta. 2010;1801(3):289-98.

40. Wang CY, Ou HY, Chen MF, Chang TC, Chang CJ. Enigmatic ectopic fat: prevalence of nonalcoholic fatty pancreas disease and its associated factors in a Chinese population. J Am Heart Assoc. 2014;3(1):e000297.

41. Della Corte C, Mosca A, Majo F, Lucidi V, Panera N, Giglioni E, et al. Nonalcoholic fatty pancreas disease and nonalcoholic fatty liver disease: more than ectopic fat. Clin Endocrinol (Oxf). 2015;83(5):656-62.

42. Saisho Y, Butler AE, Meier JJ, Monchamp T, Allen-Auerbach M, Rizza RA, et al. Pancreas volumes in humans from birth to age one hundred taking into account sex, obesity, and presence of type-2 diabetes. Clin Anat. 2007;20(8):933-42.

43. van der Zijl NJ, Goossens GH, Moors CC, van Raalte DH, Muskiet MH, Pouwels PJ, et al. Ectopic fat storage in the pancreas, liver, and abdominal fat depots: impact on beta-cell function in individuals with impaired glucose metabolism. J Clin Endocrinol Metab. 2011;96(2):459-67.

44. Dupont C, Sellier N, Chochillon C, Gobert JG, Helardot PG, Kalifa G. Pancreatic lipomatosis and duodenal stenosis or atresia in children. J Pediatr. 1989;115(4):603-5.

45. Lozano M, Navarro S, Pérez-Ayuso R, Llach J, Ayuso C, Guevara MC, et al. Lipomatosis of the pancreas: an unusual cause of massive steatorrhea. Pancreas. 1988;3(5):580-2.

46. Midiri M, Lo Casto A, Sparacia G, D’Angelo P, Malizia R, Finazzo M, et al. MR imaging of pancreatic changes in patients with transfusion-dependent beta-thalassemia major. AJR Am J Roentgenol. 1999;173(1):187-92.

47. So CB, Cooperberg PL, Gibney RG, Bogoch A. Sonographic findings in pancreatic lipomatosis. AJR Am J Roentgenol. 1987;149:67-8.

48. Aubert A, Gornet JM, Hammel P, Lévy P, O’Toole D, Ruszniewski P,et al. Diffuse primary fat replacement of the pancreas: an unusual cause of steatorrhea [French]. Gastroenterol Clin Biol. 2007;31(3):303-6.

49. Khatua B, El-Kurdi B, Singh V. Obesity and pancreatitis. Curr Opin Gastroenterol. 2017;33(5):374-82.

50. Taguchi M, Kubo T, Yamamoto M, Muramatsu K, Yasunaga H, Horiguchi H, et al. Body mass index influences the outcome of acute pancreatitis: an analysis based on the Japanese administrative database. Pancreas. 2014;43(6):863-6.

51. Zyromski NJ, Mathur A, Pitt HA, Lu D, Gripe JT, Walker JJ, et al. A murine model of obesity implicates the adipokine milieu in the pathogenesis of severe acute pancreatitis. Am J Physiol Gastrointest Liver Physiol. 2008;295(3):G552-8.

52. Stolzenberg-Solomon RZ, Adams K, Leitzmann M, Schairer C, Michaud DS, Hollenbeck A, et al. Adiposity, physical activity, and pancreatic cancer in the National Institutes of Health-AARP Diet and Health Cohort. Am J Epidemiol. 2008;167(5):586-97. 
53. Roberts DL, Dive C, Renehan AG. Biological mechanisms linking obesity and cancer risk: new perspectives. Annu Rev Med. 2010;61:301-16.

54. Matsuda M, Shimomura I. Increased oxidative stress in obesity: implications for metabolic syndrome, diabetes, hypertension, dyslipidemia, atherosclerosis, and cancer. Obes Res Clin Pract. 2013;7:e330-41.

55. Hori M, Takahashi M, Hiraoka N, Yamaji T, Mutoh M, Ishigamori R, et al. Association of pancreatic fatty infiltration with pancreatic ductal adenocarcinoma. Clin Transl Gastroenterol. 2014;5:e53.

56. Mathur A, Zyromski NJ, Pitt HA, Al-Azzawi H, Walker JJ, Saxena R, et al. Pancreatic steatosis promotes dissemination and lethality of pancreatic cancer. J Am Coll Surg. 2009;208(5):989-94; discussion 994-6.

57. Yang DM, Kim HC, Ryu JK, Joo KR, Ahn KJ. Sonographic appearance of focal fatty infiltration of the pancreas. J Clin Ultrasound. 2010;38(1):45-7.

58. Mathur A, Pitt HA, Marine M, Saxena R, Schmidt CM, Howard TJ, et al. Fatty pancreas: a factor in postoperative pancreatic fistula. Ann Surg. 2007;246(6):1058-64.

59. Gaujoux S, Cortes A, Couvelard A, Noullet S, Clavel L, Rebours V, et al. Fatty pancreas and increased body mass index are risk factors of pancreatic fistula after pancreaticoduodenectomy. Surgery. 2010;148(1):15-23.

60. Lee SE, Jang JY, Lim CS, Kang MJ, Kim SH, Kim MA, et al. Measurement of pancreatic fat by magnetic resonance imaging: predicting the occurrence of pancreatic fistula after pancreatoduodenectomy. Ann. Surg. 2010;251(5):932-6.

61. Rosso E, Casnedi S, Pessaux P, Oussoultzoglou E, Panaro F, Mahfud M, et al. The role of "fatty pancreas" and of BMI in the occurrence of pancreatic fistula after pancreaticoduodenectomy. J Gastrointest Surg. 2009;13(10):1845-51. 